Radiologe 2019 - 59:359-368 https://doi.org/10.1007/s00117-019-0511-z Online publiziert: 18. März 2019

(C) Der/die Autor(en) 2019
L. Schöckel $\cdot$ T. Balzer ${ }^{2} \cdot$ H. Pietsch ${ }^{3}$

'Pharmaceuticals Division, Medical \& Clinical Affairs Radiology, Bayer AG, Berlin, Deutschland ${ }^{2}$ Pharmaceuticals, Medical \& Clinical Affairs Radiology, Bayer U.S. LLC, Whippany, USA

${ }^{3}$ Research \& Development, Pharmaceuticals, MR and CT Contrast Media Research, Bayer AG, Berlin, Deutschland

\title{
Erhöhte Signalintensitäten und Gadolinium-Werte im Gehirn nach Gabe Gadolinium-haltiger MR-Kontrastmittel
}

\section{Klinische Beobachtungen und Ergebnisse aus präklinischer Forschung}

\begin{abstract}
Klinische Studien zeigen einen Zusammenhang zwischen der wiederholten Gabe Gadolinium(Gd)haltiger Kontrastmittel und einer erhöhten Signalintensität auf nativen, T1-gewichteten Aufnahmen in bestimmten Hirnarealen. Nach einem evidenzbasierten Risikobewertungsverfahren hat die Europäische Kommission im November 2017 die weitere Anwendung von makrozyklischen Kontrastmitteln bestätigt und den Gebrauch von linearen Kontrastmitteln, mit Ausnahme der Leberbildgebung mit Gadoxetsäure (Primovist ${ }^{\circledR}$ ) und Gadobensäure (MultiHance ${ }^{\circledR}$ ) sowie der intraartikulären Anwendung von Gadopentetsäure (Magnevist ${ }^{\circledR}$ ), untersagt. Es gibt derzeit keine Evidenz, dass Gadolinium im Gehirn bei Patienten Schäden verursacht.
\end{abstract}

Seit 30 Jahren sind Gadolinium-haltige MR-Kontrastmittel (KM) aus der Diagnostik nicht wegzudenken und haben weltweit in mehr als 500 Mio. Anwendungen ihre klinische Bedeutung, sowohl für den Patienten als auch für den Radiologen, belegt. Durch ihre gute allgemeine Verträglichkeit gelten Gd-haltige MR-Kontrastmittel als sehr sichere Arzneimittel.
Im November 2017 hat die Europäische Kommission (EK), einer Empfehlung der Europäischen Arzneimittelbehörde (EMA) folgend, den Einsatz einiger Gd-haltiger KM innerhalb der EU untersagt (- Tab. 1). Das Ruhen der Zulassung betrifft die linearen KM Gadopentetsäure (Magnevist ${ }^{\circledR}$ ), Gadodiamid (Omniscan ${ }^{\mathrm{m}}$ ), Gadoversetamid $\left(\right.$ Optimark $\left.^{\circledR}\right)$ und Gadobensäure (MultiHance $^{\circledR}$ ) für alle Anwendungen mit Ausnahme der Leberindikation von Gadobensäure und der intraartikulären Anwendung von Gadopentetsäure. Im Gegensatz dazu haben die Zulassungen des linearen leberspezifischen KM Gadoxetsäure (Primovist ${ }^{\circledR}$ ) sowie aller makrozyklischen KM (Gadotersäure [Dotarem ${ }^{\circledR}$ ], Gadobutrol [Gadovist ${ }^{\circledR}$ ] und Gadoteridol [ProHance $\left.{ }^{\circledR}\right]$ ) weiterhin uneingeschränkt Bestand. Für die Bundesrepublik Deutschland hat das Bundesinstitut für Arzneimittel und Medizinprodukte (BfArM) das Ruhen der Zulassungen am 28. Februar 2018 umgesetzt.

Kanda et al. [1] stellten erstmalig Ende 2013 einen Zusammenhang zwischen erhöhten Signalintensitäten (SI) in bestimmten Hirnarealen (Nucleus dentatus und Globus pallidus) und der wiederholten Gabe Gd-haltiger KM her. In zahlreichen weiteren Publikationen wurden diese Beobachtungen bestätigt und deutliche Unterschiede zwischen den Substanzklassen der linearen und der makrozyklischen KM belegt, jedoch ist die klinische Relevanz der beschriebenen Befunde bis zum heutigen Zeitpunkt unklar.

Die EMA/EK bezeichnet daher ihre Entscheidung im Nov 2017 auch als (aus dem Vorsorgeprinzip folgende) „precautionary measure" und betont, dass derzeit keine negativen Auswirkungen auf die Gesundheit der Patienten im Zusammenhang mit der Anwendung von linearen oder makrozyklischen KM bekannt seien.

Gesundheitsbehörden, wie z.B. die US-amerikanische (FDA), kanadische (HC) oder die japanische (PMDA), haben andere Schlussfolgerungen als die EMA/EK getroffen. Es wurden ausführlichere Ergänzungen der Packungsbeilagen eingeführt. In Japan wurden die linearen $\mathrm{KM}$ als "second line option“ deklariert. In den USA wurden klinische und präklinische Studien initiiert. Der grundsätzliche Nutzen aller Gd-haltigen KM wird allerdings nicht in Frage gestellt. Daher ist der Anwendungsbereich (Indikationen) der linearen KM bis dato nicht eingeschränkt.

Die Gründe, die zu diesen weitreichenden Beschlüssen der EK und den daraus resultierenden Gebrauchsänderun- 
Tab. 1 Übersicht des Durchführungsbeschlusses der Europäischen Kommission für die in der EU zugelassenen Gd-haltigen Kontrastmittel

\begin{tabular}{|c|c|c|}
\hline & Wirkstoff (Handelsname) & Zulassungsstatus \\
\hline \multirow{6}{*}{$\begin{array}{l}\text { Lineare } \\
\text { Gadolinium-haltige } \\
\text { MR-Kontrastmittel }\end{array}$} & Gadopentetsäure (Magnevist $\left.{ }^{\circledR}\right)$ i.v. ${ }^{a}$ & Ruhen der Zulassung \\
\hline & $\begin{array}{l}\text { Gadopentetsäure (Magnevist }{ }^{\circledR} \text { ) } \\
2 \mathrm{mmol} / /^{\mathrm{a}}\end{array}$ & $\begin{array}{l}\text { Aufrechterhaltung der Zulassung für } \\
\text { die intraartikuläre Anwendung }\end{array}$ \\
\hline & Gadobensäure (MultiHance ${ }^{\circledR}$ ) i.v. & $\begin{array}{l}\text { Einschränkung der Zulassung auf } \\
\text { die MRT Leberbildgebung }\end{array}$ \\
\hline & Gadodiamid $\left(\right.$ Omniscan $\left.^{\mathrm{TM}}\right)$ & Ruhen der Zulassung \\
\hline & Gadoversetamid (OptiMark ${ }^{\circledR}$ ) i.v. & Ruhen der Zulassung \\
\hline & Gadoxetsäure (Primovist ${ }^{\circledR}$ ) i.v. & $\begin{array}{l}\text { Aufrechterhaltung der Zulassung für } \\
\text { die MRT Leberbildgebung }\end{array}$ \\
\hline \multirow{3}{*}{$\begin{array}{l}\text { Makrozyklische } \\
\text { Gadolinium-haltige } \\
\text { MR-Kontrastmittel }\end{array}$} & $\begin{array}{l}\text { Gadotersäure }\left(\text { Dotarem }^{\circledR}\right) \text { i.v. und } \\
2 \mathrm{mmol} / \mathrm{l}^{\mathrm{b}}\end{array}$ & $\begin{array}{l}\text { Aufrechterhaltung der Zulassung in } \\
\text { allen Indikationen }\end{array}$ \\
\hline & Gadobutrol (Gadovist $\left.{ }^{\circledR}\right)$ i.v. & $\begin{array}{l}\text { Aufrechterhaltung der Zulassung in } \\
\text { allen Indikationen }\end{array}$ \\
\hline & Gadoteridol (ProHance ${ }^{\circledR}$ ) i.v. & $\begin{array}{l}\text { Aufrechterhaltung der Zulassung in } \\
\text { allen Indikationen }\end{array}$ \\
\hline \multicolumn{3}{|c|}{ 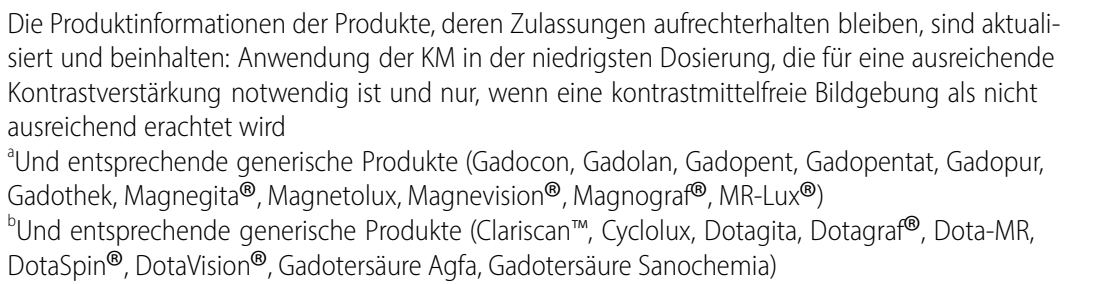 } \\
\hline
\end{tabular}

gen geführt haben, werden nachfolgend diskutiert.

\section{Klinische Beobachtungen}

\section{Bildgebungsstudien}

Erhöhte Signalintensitäten (SI) in bestimmten Hirnregionen, wie Nucleus dentatus (ND) und Globus pallidus (GP) auf nativen, T1-gewichteten (T1w) MR-Aufnahmen werden seit Langem beobachtet. In der Vergangenheit wurden diese mit Erkrankungen, wie multipler Sklerose (MS) oder Stoffwechselstörungen (Eisen- oder Manganablagerungen), Leberdysfunktion oder als Folge von Strahlentherapie in Verbindung gebracht und erklärt. Seit der Veröffentlichung von Kanda et al. [1] wurden zahlreiche wissenschaftliche Untersuchungen initiiert, um genauer zu untersuchen, ob der Effekt für alle KM gleichermaßen zu beobachten ist und inwieweit auch andere Faktoren für das Phänomen zumindest mit verantwortlich sind.

Mittlerweile (Stand:Okt 2018) werden in mehr als 50 klinische Publikationen erhöhte SI-Werte insbesondere nach wie- derholter intravenöser Gabe linearer KM beschrieben. Fast alle Studien sind retrospektiv und haben entsprechende Limitationen, wie z.B. die nicht lückenlos dokumentierte Patientenhistorie, frühere KM-Gaben, Unterschiede in der MRBildakquise (Hersteller, Feldstärke, Sequenzen usw.).

In alle Studien mit linearen KM wird eine sichtbare SI-Erhöhung im ND und GP berichtet, vorausgesetzt, eine Gesamtdosis von etwa 5-6 Standarddosis-Applikationen $(0,1 \mathrm{mmol} \mathrm{Gd} / \mathrm{kg})$ wurde überschritten (• Abb. 1; [1-5]). Unterschiede innerhalb der linearen $\mathrm{KM}$, die für die breite Anwendung (ZNS, Ganzkörper) zugelassen waren, sind nicht festzustellen. Für Gadoxetsäure $\left(\right.$ Primovist $\left.^{\circledR}\right)$, ein leberspezifisches, ionisch lineares KM, das in einer $75 \%$ geringeren Dosis $(\hat{=} 0,025 \mathrm{mmol}$ $\mathrm{Gd} / \mathrm{kg}$ ) als alle anderen KM (Ausnahme Leberbildgebung mit Gadobensäure [MultiHance ${ }^{\circledR}$ ]; 0,05 mmol Gd/kg) appliziert wird, existieren derzeit drei klinische Studien. Zwei dieser Studien zeigen keine SI-Zunahme im ND nach bis zu 15 oder 18 Applikationen [6, 7]. Eine dritte Studie berichtet über eine
SI-Erhöhung im ND bei Patienten, die 11-37 Injektionen erhielten, während bei Patienten mit weniger als 10 Gadoxetsäure (Primovist ${ }^{\circledR}$ ) Injektionen keine erhöhte SI beobachtet wurde [8].

In mehr als 24 klinischen Studien, überwiegend mit den Makrozyklen Gadotersäure $\left(\right.$ Dotarem $^{\circledR}$ ) und Gadobutrol $\left(\right.$ Gadovist $\left.^{\circledR}\right)$, wurde weder eine sichtbare noch eine statistisch signifikant messbare SI-Erhöhung im Gehirn von Patienten, teilweise nach bis zu 50 Injektionen berichtet [3, 9-11]. In 4 Studien werden Effekte nach Makrozyklen dargestellt [12-15]. Zwei Studien davon messen für einen Teil der Patienten eine erhöhte SI, jedoch waren diese auf den MR-Aufnahmen nicht sichtbar [12, 13]. Diese Publikationen haben deutliche Limitationen, die auch öffentlich kontrovers diskutiert wurden. Die Befunde sind daher nicht allgemein akzeptiert [16-21].

\section{Ergebnisse aus Untersuchungen von Gewebeproben aus Autopsien}

Neben den MR-Studien sind bis heute 8 „Gewebestudien“ veröffentlicht worden, welche Gd-Konzentrationen mittels massenspektroskopischer Messung im Hirngewebe verstorbener Patienten untersucht haben [22-29]. Die Aussagekraft der Studien ist zum einen aufgrund der sehr geringen Patientenzahlen, zum anderen durch fehlende Vergleichbarkeit (z. B. unterschiedliche Zeitintervalle zwischen letzter KM-Gabe und BiopsieGewinnung) limitiert, zumindest was quantitative Vergleiche anbelangt. Aus den Gd-Konzentrationsbestimmungen geht hervor, dass minimale Gd-Mengen im Hirngewebe nach Gabe aller KM zu detektieren waren. Grundsätzlich scheinen die Gd-Konzentrationen nach Gabe linearer KM höher zu sein, wobei die gemessenen Gd-Konzentrationen im Gehirn zu gering sind, um die SIAnstiege zu erklären.

Wenige Studien untersuchten GdKonzentrationen in anderen Organen, vorzugsweise in Haut und Knochen [26, 30-32]. Murata et al. [26] veröffentlichte postmortale Gd-Konzentrationen im Knochen bei 8 Patienten, welche lineare und makrozyklische KM erhielten. Die gemessenen Gd-Konzentrationen bei 
allen Patienten waren um ein Mehrfaches höher als die Konzentrationen im Gehirn. Da das Zeitintervall zwischen KM-Gabe und Gewebe-Entnahme stark variiert, ist kein quantitativer Vergleich der Gd-Konzentrationen zwischen den verschiedenen KM möglich. Des Weiteren können vorherige KM-Gaben nicht ausgeschlossen werden.

Letztlich ist jedoch davon auszugehen, dass Spuren von Gd nach der Gabe aller Gd-haltigen KM sowohl im Gehirn als auch in anderen Organen (Knochen, Haut) z.T. auch Jahre nach der letzten bekannten KM-Gabe detektierbar sein können. Dies deutet auf eine sehr langsame Gd-Ausscheidung oder eine mögliche Einlagerung von $\mathrm{Gd}$ in veränderter Form hin.

Auch in diesen Studien wurde jedoch kein Zusammenhang zwischen klinischen Symptomen, erhöhten SI und den Gd-Werten in verschiedenen Hirnarealen oder anderen Organen berichtet.

\section{Stabilitätsunterschiede der Gd-haltigen KM als Ursache für erhöhte SI?}

Mehrere klinische Veröffentlichungen weisen darauf hin, dass Unterschiede in der Stabilität der Gd-haltigen KM die erhöhten SI im Gehirn erklären könnten. Präklinische Studien unterstützen diese Annahme.

Die Stabilität der Gd-Komplexe ist abhängig von ihrer chemischen Struktur. Sie lassen sich in makrozyklische, von DOTA $(1,4,7,10$-Tetraazacyclododecantetraessigsäure) abgeleitete Komplexe und lineare, von DTPA (Diethylentriaminpentaessigsäure) abgeleitete Komplexe einteilen. Sie sind entweder ungeladen, wodurch neutrale Komplexe entstehen, oder der Gd-Komplex trägt 1-3 negative Ladungen, wodurch ionische Komplexe gebildet werden.

Zwei Parameter beschreiben die Stabilität der Komplexe: die thermodynamische Stabilität des Komplexes und die kinetische Stabilität, welche die Kinetik der Gd-Freisetzung unter physiologischen Bedingungen beschreibt.

Makrozyklische KM sind unter physiologischen Bedingungen kinetisch inert, d.h. keine messbaren Gd-Mengen

Radiologe 2019·59:359-368 https://doi.org/10.1007/s00117-019-0511-z

(c) Der/die Autor(en) 2019

L. Schöckel · T. Balzer · H. Pietsch

Erhöhte Signalintensitäten und Gadolinium-Werte im Gehirn nach Gabe Gadolinium-haltiger MR-Kontrastmittel. Klinische Beobachtungen und Ergebnisse aus präklinischer Forschung

\section{Zusammenfassung}

Hintergrund. In zahlreichen klinischen MRTStudien wird ein Zusammenhang zwischen der wiederholten Gabe (linearer) Gadoliniumhaltiger MR-Kontrastmittel und einer erhöhten Signalintensität in bestimmten Hirnarealen diskutiert. Im November 2017 hat die Europäische Kommission die Anwendung einiger dieser Kontrastmittel untersagt.

Ziel der Arbeit. Die Gründe für diese Entscheidung werden dargestellt und diskutiert.

Material und Methoden. Die regulatorischen Beschlüsse werden ausgewertet und klinische und präklinische Forschungsarbeiten diskutiert.

Ergebnisse. Unterschiede in der Struktur und Stabilität der Gadolinium-haltigen
MR-Kontrastmittelmoleküle erklären die beobachteten erhöhten Signalintensitäten in einzelne Hirnbereichen (z. B. Nucleus dentatus) nach mehrmaliger Injektion linearer Kontrastmittel. Dieses Phänomen wurde nicht nach wiederholter Gabe makrozyklischer Kontrastmittel beobachtet. Präklinische Studien konnten diese Ergebnisse bestätigen. Diskussion. Bis heute sind keine klinischen Symptome berichtet worden, die durch erhöhte Signalintensitäten oder GadoliniumWerte im Gehirn verursacht wurden.

Schlüsselwörter

Gehirn · Nucleus dentatus - Globus pallidus . Klinischer Nutzen · Stabilität Gd-haltiger KM

\section{Increased signal intensities and gadolinium levels in the brain after administration of gadolinium-based MR contrast agents. Clinical observations and results from preclinical research}

\section{Abstract}

Background. Numerous clinical MRI studies have been published that describe an association between the repeated administration of (linear) gadolinium-based contrast agents and increased signal intensity in certain brain areas. In November 2017, the European Commission suspended the use of some of these contrast agents.

Objectives. The background for this decision, both regulatory and scientific, are presented and discussed.

Materials and methods. The regulatory decisions are evaluated and the clinical and preclinical literature is discussed.

Results. Differences in the structure and stability of gadolinium-based contrast agent molecules explain the observed increased signal intensities in individual brain regions (e. g. dentate nucleus) after administration of multiple doses of linear contrast agents. This phenomenon was not observed after administration of multiple doses of macrocyclic contrast agents. Preclinical studies have confirmed these results. Conclusion. To date, no clinical symptoms have been confirmed to be associated with the increased signal intensity or gadolinium presence in the brain.

Keywords

Brain · Dentate nucleus · Globus pallidus . Clinical benefits - Stability of Gd-based contrast agents werden freigesetzt. Grund dafür ist die Struktur des Moleküls, d.h. das $\mathrm{Gd}^{3+}$ Ion wird in einer Art Käfig festgehalten (-Abb. 2). Dadurch ist eine mögliche Freisetzung von Gd, unabhängig von der thermodynamischen Stabilität der Makrozyklen, extrem langsam und unter physiologischen Bedingungen in vivo nicht relevant [34].
Um die Stabilität der linearen KM zu beschreiben, ist die thermodynamische Stabilitätskonstante der wichtigere Parameter. Die thermodynamischen Stabilitätskonstanten beschreiben das Gleichgewicht zwischen der Konzentration des Gd-Komplexes auf der einen und der Konzentration des freien $\mathrm{Gd}^{3+}$-Ions und des freien Liganden auf der anderen Seite. Ein ionisch linearer Gd-Komplex hat ei- 

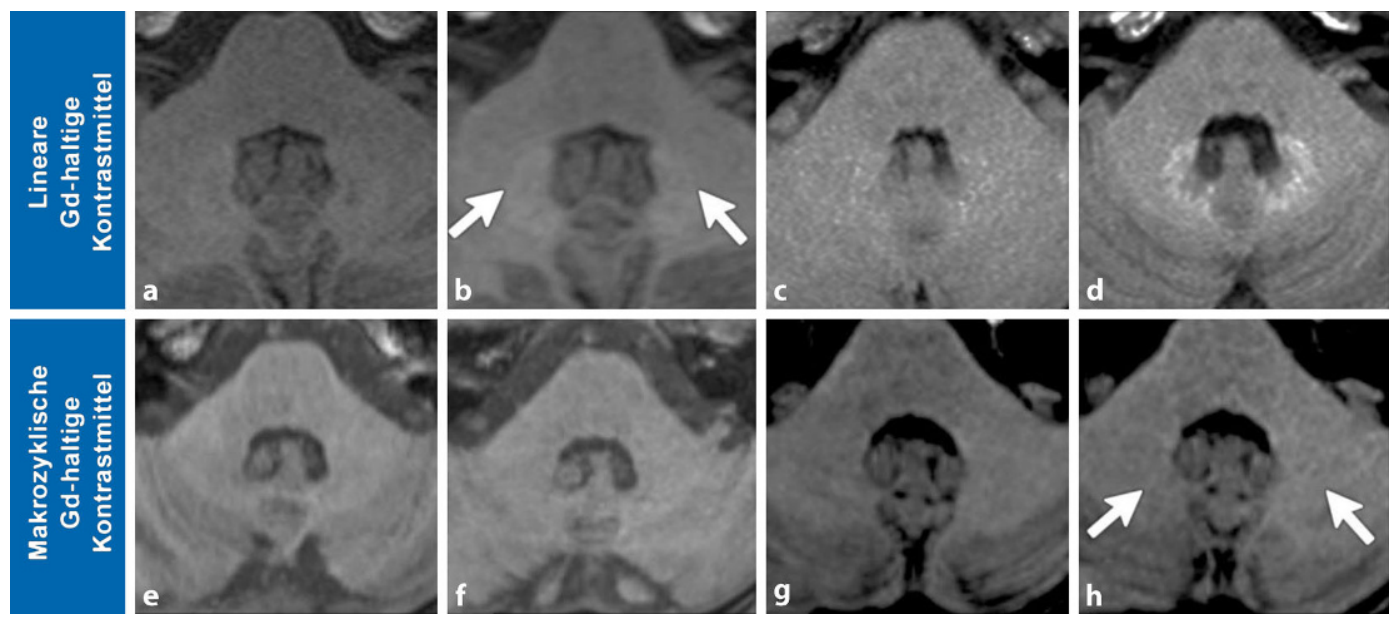

Abb. 1 ॥ Zunahme der Signalintensität auf nativen T1w-Aufnahmen im Nucleus dentatus nach Verabreichung von linearen Gd-haltigen MR-Kontrastmitteln (a-d), jedoch nicht nach der Gabe makrozyklischer Kontrastmittel (e-h). a, b Native T1w-Aufnahmen vor (a) und nach (b) dem 6.Scan mit Gadopentetsäure (Magnevist ${ }^{\circledR}$ ). c, d Native T1 w-Aufnahmen vor (c) und nach (d) dem 16. Scan mit Gadobensäure (MultiHance ${ }^{\circledR}$ ). e, f Native T1w-Aufnahmen vor (e) und nach (f) dem 19. Scan mit Gadobutrol (Gadovist ${ }^{\circledR}$ ). g, h Native T1w-Aufnahmen vor (g) und nach (h) dem 6. Scan mit Gadotersäure (Dotarem $\left.{ }^{\circledR}\right)$. (a, b, g, h Aus [2]. c-f Aus [3] mit freundlicher Genehmigung)

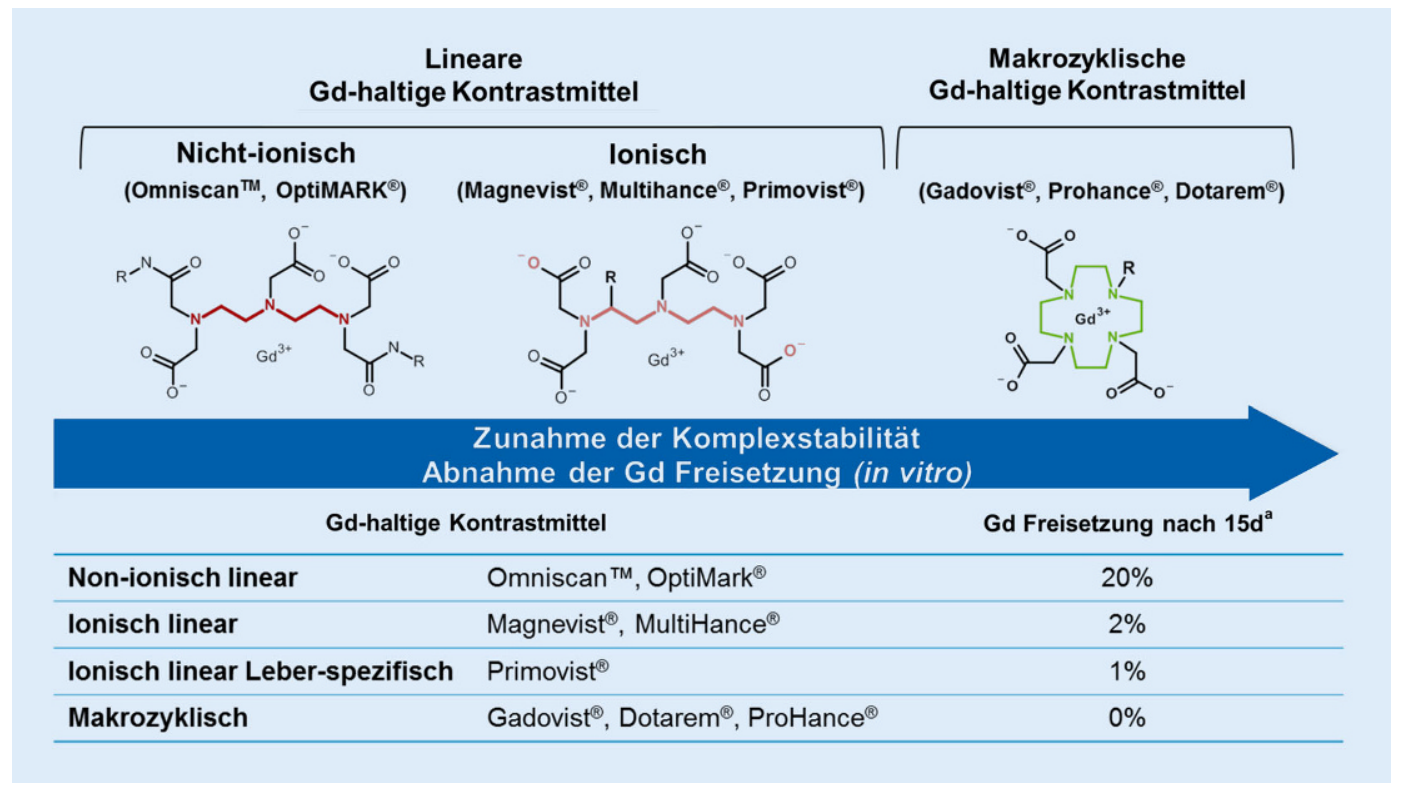

Abb. $2<$ Freisetzung von Gadolinium aus dem GdKomplex in Humanserum. $\mathrm{a}=$ gemessen in humanen Serum bei $37^{\circ} \mathrm{C}$ (Aus [33] mit freundlicher Genehmigung)

ne stärkere Bindung des $\mathrm{Gd}^{3+}$-Ions als ein neutraler, also ein nichtionisch linearer Gd-Komplex. Lineare KM sind deutlich instabiler als makrozyklische KM, d.h. ein Gleichgewicht kann innerhalb weniger Minuten bis Stunden erreicht werden.

Die thermodynamischen Stabilitätskonstanten können angesichts der extrem langen Trennungshalbwertzeit (extrapoliert $>1000$ Jahre bei $\mathrm{pH} 7,4$ ) bei makrozyklischen KM vernachlässigt werden. Unter Berücksichtigung beider Parameter, der thermodynamischen und kinetischen Stabilität, lässt sich folgende Rang- folge der Stabilität von Gd-haltigen KM unter physiologischen Bedingungen aufstellen: makrozyklische KM > ionisch lineare $\mathrm{KM}>$ nichtionisch lineare $\mathrm{KM}$.

\section{Präklinische Ergebnisse}

Für das Verständnis der vorliegenden Befunde aus den Bildgebungs- und „Gewebestudien“ sind folgende Punkte von besonderer Bedeutung:

- Hat die Nierenfunktion einen Einfluss auf die erhöhten SI- und Gd-Werte?
- Wie gelangen Gd-haltige KM nach i.v.-Injektion ins Gehirn?

- In welcher molekularen Form liegt Gd im Gehirn vor?

- Führt Gd im Gehirn zu histopathologischen Veränderungen?

- Bleiben die Gd-Konzentrationen konstant, oder nehmen sie im Lauf der Zeit $a b$ ?

Um mögliche Effekte, die mit der Gabe Gd-haltiger KM assoziiert werden, untersuchen zu können, wurden zahlreiche in vitro und in vivo Modelle etabliert. Be- 


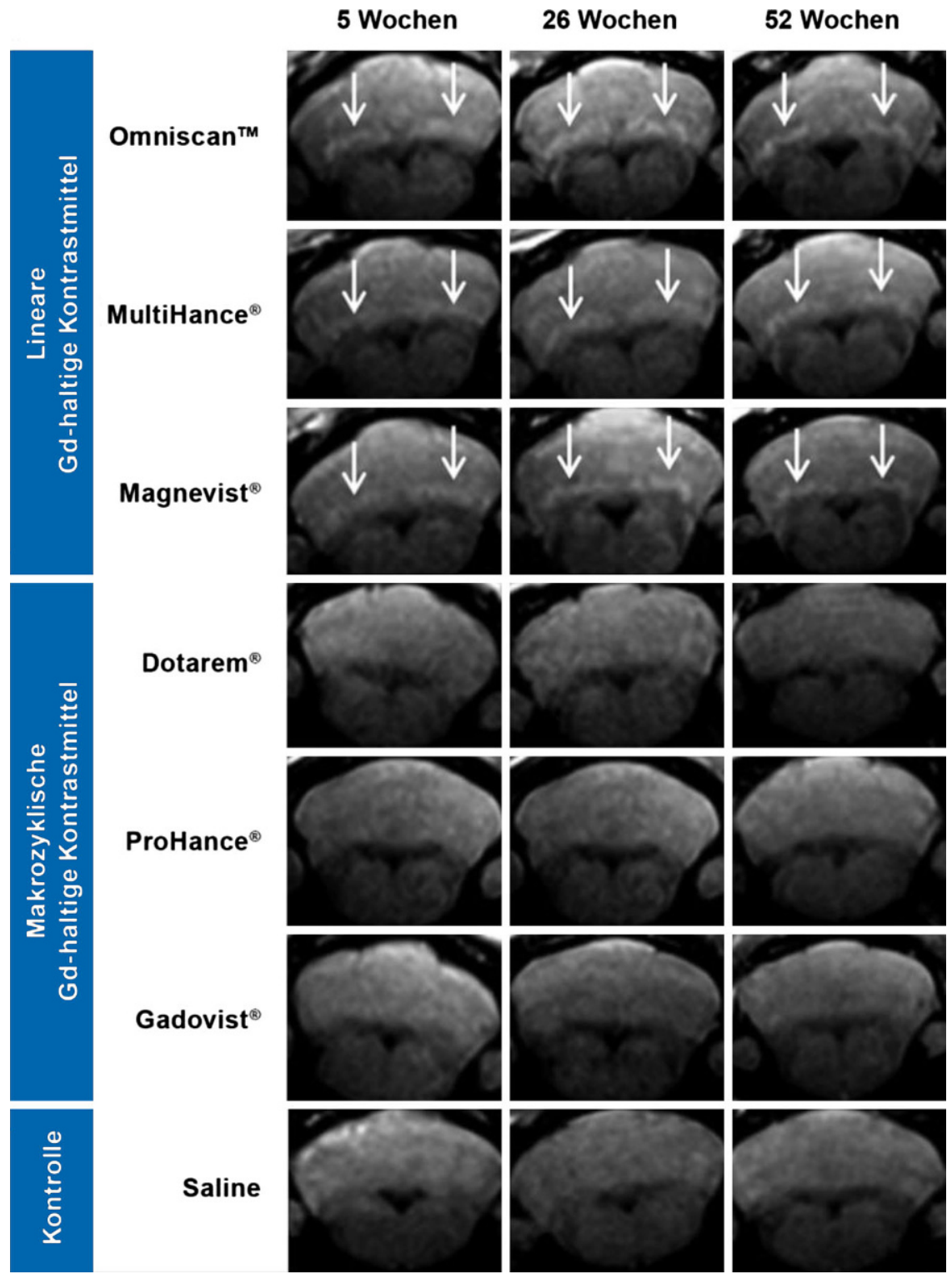

Abb. $3 \Delta$ T1w MR-Aufnahmen des Kleinhirns mit den zerebellaren Nuklei zu verschiedenen Zeitpunkten (5, 26, 52 Wochen p.i.). WeißePfeile SI-Erhöhung. (Aus [40] mit freundlicher Genehmigung)

reits bei der Aufklärung der Ätiologie der nephrogenen systemischen Fibrose (NSF) waren die Ergebnisse aus tierexperimentellen Untersuchungen entscheidend zum besseren Verständnis der Ursachen und Zusammenhänge [35].

\section{Reproduktion der Bildbefunde im Tiermodell}

\section{Erhöhte SI im Gehirn ausschließlich nach Gabe linearer Kontrastmittel} Schwerpunkt der ersten präklinischen Studien waren T1w MRT-Untersuchungen an Ratten, um die Reproduzierbarkeit der Befunde am Patienten im Tiermodell zu überprüfen. Insgesamt haben vier präklinische Studien einheitlich zeigen können, dass ausschließlich nach wiederholter Gabe linearer KM erhöhte SI im Gehirn gefunden wurden [36-39]. Für die Makrozyklen Gadobutrol (Gadovist ${ }^{\circledR}$ ), Gadoteridol (ProHance ${ }^{\circledR}$ ) oder Gadotersäure $\left(\right.$ Dotarem $\left.^{\circledR}\right)$ konnte das nicht gezeigt werden. In einer weiteren Studie wurden die Versuchstiere bis $1 \mathrm{Jahr}$ nach letztmaliger KM-Gabe untersucht. In der T1w MRT wurde durchgängig und unverändert, über den gesamten Untersuchungszeitraum erhöhte SI in den tiefen zerebralen Kernen für Gadodiamid (Omniscan $\left.{ }^{\mathrm{mm}}\right)$, Gadopentetsäure $\left(\right.$ Magnevist ${ }^{\circledR}$ ) und Gadobensäure (MultiHance $^{\circledR}$ ) gefunden, jedoch nicht für die Makrozyklen, wie Gadobutrol $\left(\right.$ Gadovist $\left.^{\circledR}\right)$, Gadoteridol (ProHance ${ }^{\circledR}$ ) oder Gadotersäure (Dotarem ${ }^{\circledR}$; $\bullet$ Abb. 3; [40]). Die gleiche Studie wurde mit Gadodiamid $\left(\mathrm{Omniscan}^{\mathrm{nx}}\right)$ und Gadotersäure $\left(\right.$ Dotarem $\left.^{\circledR}\right)$ durchgeführt, wobei ausschließlich Gadodiamid (Omniscan" bis ein Jahr nach letzter Injektion einen persistenten Ansteig der SI auswies [41].

Kontrovers zu diesen Studien ist eine präklinische Untersuchung von McDonald et al. Hier wurde 3 Tage nach letzter Injektion erhöhte SI nach Gadodiamid (Omniscan ${ }^{\mathrm{m}}$ ) und Gadobensäure (MultiHance ${ }^{\circledR}$ ), aber auch in geringerem Maße - nach Gadobutrol $\left(\right.$ Gadovist $^{\circledR}$ ) ermittelt [42]. Aus den vorgestellten Daten und technischen Details ihrer Generierung muss die Validität der Daten jedoch infrage gestellt werden [43, 44]. Beispielhaft sei erwähnt, dass nicht im tiefen zerebellaren Nuklei des Gehirns, sondern ein Bereich im 4. Ventrikel (Plexus choroideus) gemessen wurde [44].

\section{Hat die Nierenfunktion einen Einfluss auf die erhöhten SI und Gd-Werte?}

Rasschaert et al. führten MRT-Untersuchungen an 5/6 nephrektomierten Ratten durch. Nach Gabe von Gadodiamid (Omniscan ${ }^{\mathrm{mm}}$ ) waren die SI im Gehirn und die Gd-Konzentrationen in allen Geweben signifikant höher in Tieren mit eingeschränkter Nierenfunktion im Vergleich zur Kontrolle [45]. In Mäusen mit eingeschränkter Nierenfunktion wurden nach Gadodiamid $\left(\mathrm{Omniscan}^{\mathrm{mm}}\right)$ erhöhte Gd-Konzentrationen nur zu frühen Zeitpunkten (3 Tage p.i.) in Leber, Knochen, Milz und Haut gemessen, jedoch nicht im Gehirn. Zu späten Zeitpunkten (45 Tage p.i.) waren die Konzentrationen der niereninsuffizienten und der gesunden Tiere wieder auf gleichem Niveau. Für Gadotersäure $\left(\right.$ Dotarem $\left.^{\circledR}\right)$ waren die gemessenen Konzentrationen deutlich geringer. Unterschiede zwischen nierengesunden und kranken Tieren konnten, mit Ausnahme einer höheren Gd-Konzentration 


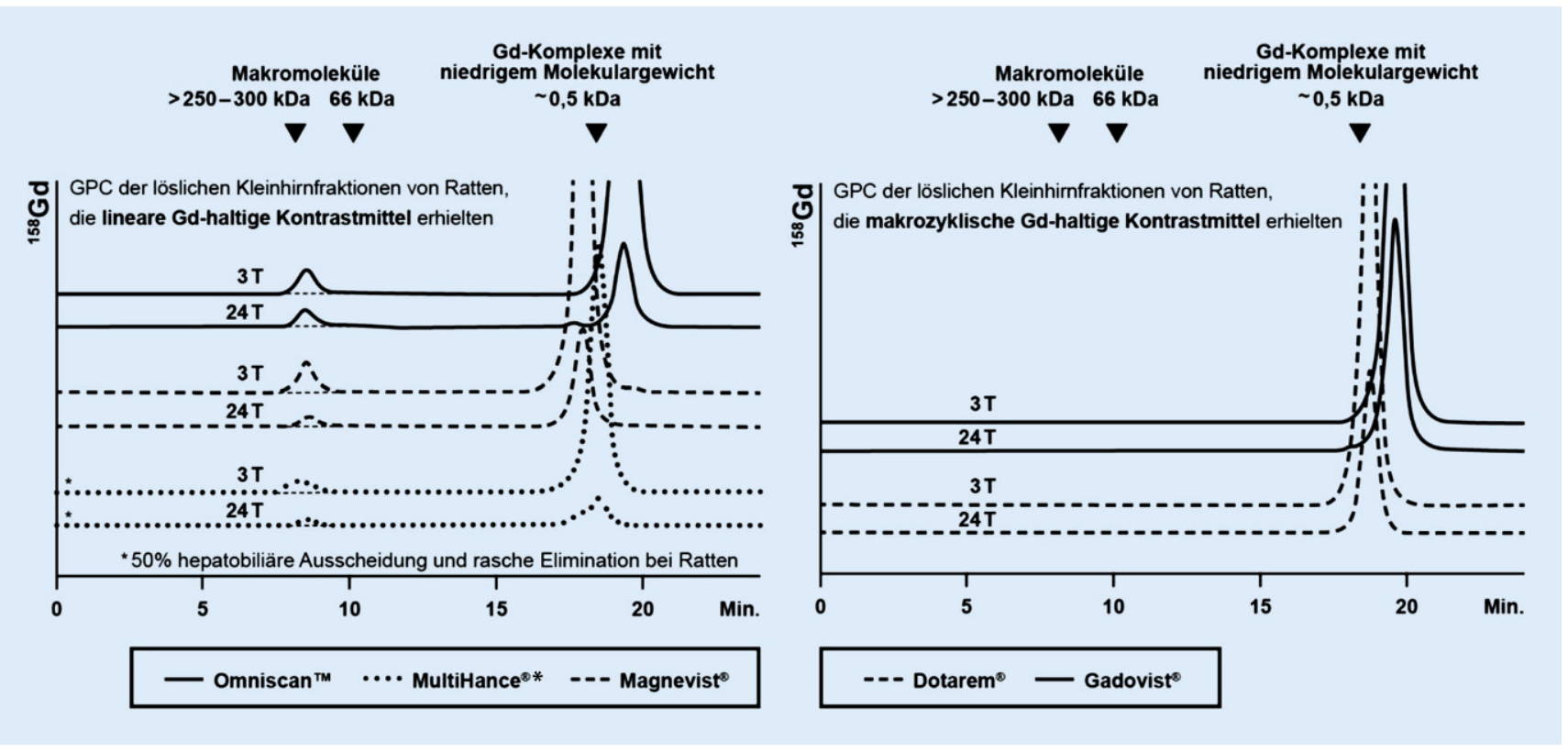

Abb. 4 a Gadolinium-spezifische Chromatogramme von Kleinhirnhomogenaten von Ratten, 3 und 24 Tage nach Gabe linearer und makrozyklischer Kontrastmittel. *Geringere Peakfläche durch schnellere Eliminierung von Gadobensäure (MultiHance ${ }^{\circledR}$ ) wegen hepatobiliärer Ausscheidung, die bei Ratten etwa 50\%, beim Menschen aber nur 3-5\% beträgt. Gd Gadolinium, GPC Gel Permeation Chromatography. (Aus [51] mit freundlicher Genehmigung)

in der Leber, nicht nachgewiesen werden [46].

\section{Wie gelangen Gd-haltige KM nach} i.v.-Injektion ins Gehirn?

Lange galt die Annahme, dass Gd-haltige KM bei intakter Blut-Hirn-Schranke nicht in den interzellulären Raum des Gehirns gelangen können. Im Tierexperiment wurde diese Fragestellung untersucht [47]. Für alle KM wurde eine vergleichbare Verteilungskinetik in die mit Zerebrospinalflüssigkeit (CSF) gefüllten Räume beobachtet. Zum frühen Zeitpunkt waren die Gd-Konzentrationen in der CSF deutlich höher als im Blut, jedoch nach $24 \mathrm{~h}$ aus der CSF fast vollständig ausgeschieden. Alle Gd-haltigen KM, unabhängig von ihrer chemischen Struktur oder physikochemischen Eigenschaft, gelangen nach i.v.-Gabe über die Gefäßgeflechte in den Ventrikeln (Plexus choroideus) in die mit CSF gefüllten Räume, verteilen sich in den Ventrikeln weiter in den subarachnoidalen Raum und mit der CSF über den Robin-Virchow-Raum in das Gehirn. Das glymphatische System, ein hirnweites Netzwerk, dessen Funktion die Ausscheidung von Metaboliten und Schadstoffen aus dem Gehirn ist, hat einen weiteren Einfluss auf die Verteilung und Ausscheidung der Gd-haltigen KM im Gehirn [48, 49]. In einer tierexperimentellen Studie wurde gezeigt, dass eine Blut-Hirn-Schranken-Störung, wie sie bei intrakraniellen Tumoren auftritt, zu keiner Erhöhung der SI im ND führt [50].

\section{In welcher molekularen Form liegt Gd im Gehirn vor?}

Gd-haltige KM sind sehr gut wasserlöslich und werden sehr schnell aus dem Körper ausgeschieden. In Ratten wurden nach Applikation von Gadobutrol $\left(\right.$ Gadovist $\left.^{\circledR}\right)$, Gadotersäure (Dotarem ${ }^{\circledR}$ ), Gadopentetsäure (Magnevist $\left.{ }^{\circledR}\right)$, Gadobensäure (MultiHance ${ }^{\circledR}$ ) und Gadodiamid $\left(\right.$ Omniscan $\left.{ }^{\mathrm{mw}}\right) 3$ Tage bzw. 24 Tage nach der letzter Injektion die Gehirne der Versuchstiere homogenisiert, fraktioniert und anschließend chromatographisch untersucht (• Abb. 4). Zu beiden Zeitpunkten waren nur noch GdSpuren der injizierten Dosis nachweisbar. Makrozyklen lagen ausschließlich in der löslichen Fraktion vor und wurden als intakter Gd-Komplex detektiert. Im Gegensatz dazu wurde bei allen, mit linearen $\mathrm{KM}$ behandelten Tieren Gd auch in der unlöslichen Gewebefraktion gefunden, vermutlich in Form von Gd-Phosphat. In der löslichen Fraktion wurden zwei Spezies identifiziert. Neben dem intakten Gd-Komplex wurde zusätzlich für alle linearen $\mathrm{KM}, \mathrm{Gd}$ detektiert, welches an Makromoleküle gebunden ist. Dieses an Makromoleküle gebundene Gd ist die Ursache für die erhöhte SI im Gehirn nach Gabe linearer KM [51].

\section{Führt Gd im Gehirn zu histopatho- logischen Veränderungen?}

Lohrke et al. haben in ihrer Studie nach mehrmaligen Injektionen von Gadodiamid $\left(\mathrm{Omniscan}^{\mathrm{Tm}}\right)$ massive Hautveränderungen festgestellt, welche histopathologisch als NSF eingestuft wurden. In den Gehirnen wurden bei allen Tieren, auch bei den Tieren mit Hautveränderungen, keinerlei Befunde festgestellt [52]. In einer weiteren Studie wurden die Tiere 20 Wochen nach der letzten KM-Gabe von Gadodiamid $\left(\right.$ Omniscan $\left.{ }^{\mathrm{m}}\right)$ untersucht. Auch hier konnten keine histologischen Veränderungen in den Gehirnen detektiert werden [53]. 


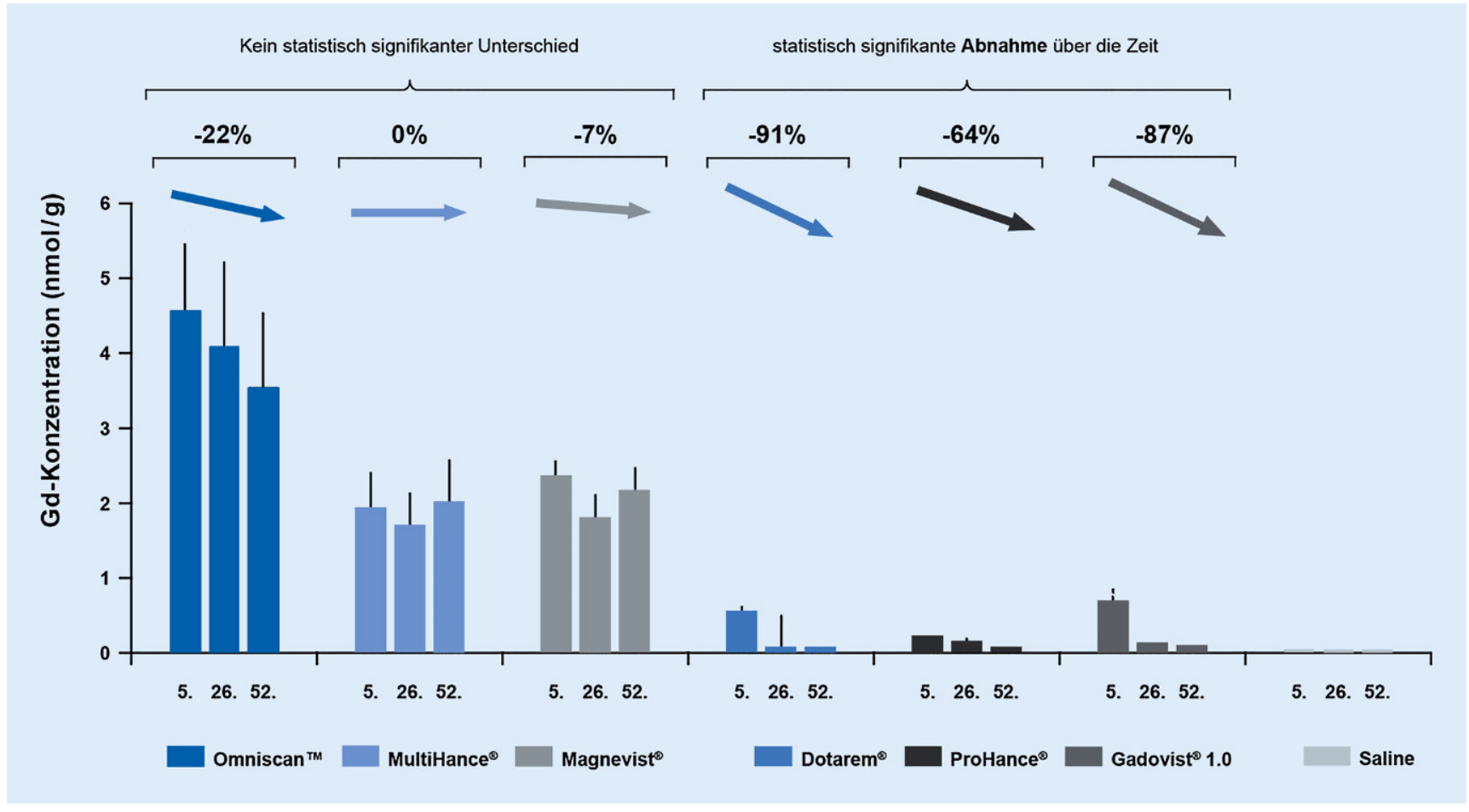

Abb. $5 \Delta$ Gadolinium-Konzentrationen vom Rattenkleinhirn, bestimmt durch ICP-MS (Massenspektrometrie mit induktiv gekoppeltem Plasma). (Aus [40] mit freundlicher Genehmigung)

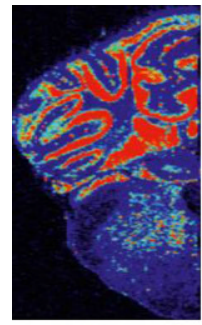

Omniscan $^{\mathrm{TM}}$

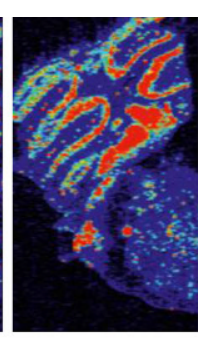

MultiHance $^{\circledast}$

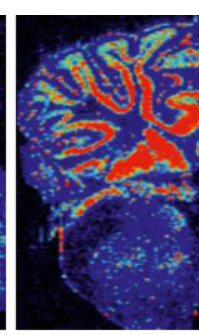

Magnevist ${ }^{\circledR}$

Min

Lineare Gd-haltige Kontrastmittel

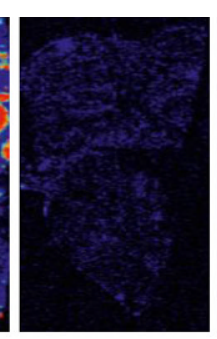

Dotarem $^{\circledast}$

Min Makrozyklische Gd-haltige Kontrastmittel

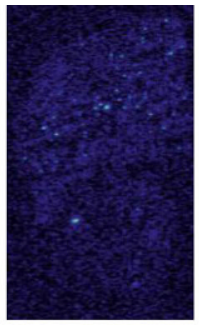

ProHance ${ }^{\circledast}$

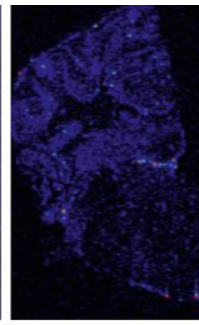

Gadovist $^{\circledast}$

$9,5 \mathrm{nmol} / \mathrm{g}$

deutlich niedriger als für Gadodiamid $\left(\right.$ Omniscan $\left.^{\mathrm{su}}\right)$. Bei den Makrozyklen waren die Gd-Konzentrationen auf einem deutlich niedrigeren Niveau (ca. 40-mal geringer). Bemerkenswert ist, dass für mindestens 1 Jahr nach der Gabe linearer KM die gemessenen Gd-Konzentrationen im Kleinhirn nahezu konstant bleiben und ab ca. 5 Wochen nach der letzten KM-Gabe keine weitere Elimination mehr erfolgt. Für Makrozyklen zeigt sich während des gesamten Beobachtungszeitraums eine kontinuierliche Elimination.

Mit der Kombination von Laserablation und Massenspektrometrie lassen

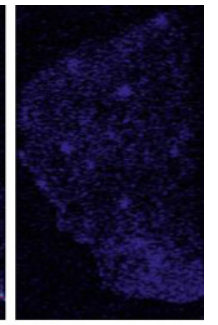

Saline

Abb. $6<$ Gadolinium-Verteilung im Gehirn von Ratten 52 Wochen nach letzter Kontrastmittel-Injektion.

Kontrolle
(Aus [40] mit freundlicher Genehmigung)

\section{Bleiben die Gd-Konzentrationen}

Von Bedeutung ist sicherlich auch, wie lange ggf. Spuren der KM im Körper und seinen Organe verbleiben bzw. ob diese langsam aber vollständig ausgeschie aur Dreifachdosis beim schen) wurden die höchsten Gd-Konvon Gadodiamid (Omniscan ${ }^{\mathrm{nx}}$ ) gemessen (• Abb. 5; [40]). Die Konzentrationen für Gadopentetsäure (Magnevist ${ }^{\circledR}$ ) und Gadobensäure (MultiHance ${ }^{\circledast}$ ) sind vergleichbar, aber für beide Substanzen sich auf histologischen Schnitten die GdVerteilung auch (semi)quantitativ darstellen. 52 Wochen nach Gabe linearer KM (Gadodiamid [Omniscan" ${ }^{\mathrm{m}}$, Gadobensäure [MulitHance ${ }^{\circledast}$ ] und Gadopentetsäure [Magnevist ${ }^{\circledast}$ ]) wurden relevante Konzentrationen von Gd, sehr spezifisch lokalisiert in den tiefen zerebralen Kernen und im Stratum granulosum nachgewiesen. Für Makrozyklen wurde kein spezifisches Trapping gefunden, und die Konzentrationen waren um ein Vielfaches geringer (๑ Abb. 6; [40]).

Zusammenfassend wurden in Tierstudien folgende wichtige Erkenntnisse gewonnen: 
- Ein potenzieller Eintrittsweg Gdhaltiger KM aus dem Blut in das Gehirn ist der Liquor (CSF).

- Erhöhte SI in verschiedenen (tiefen) Hirnarealen wurde ausschließlich nach der wiederholten Gabe linearer KM beobachtet, jedoch nicht nach Makrozyklen.

- Geringe Mengen an Gd können noch 5 Wochen nach letzter Injektion aller Gd-haltiger KM nachgewiesen werden, jedoch ist die Konzentration im Gehirn bei den linearen deutlich höher als die bei den Makrozyklen.

- Die Gd-Konzentrationen nach Gabe linearer KM bleiben über einen Beobachtungszeitraum von 5-52 Wochen nahezu konstant. Dagegen ist für makrozyklische KM eine kontinuierliche Abnahme zu erkennen.

- Die Stabilität des Gd-Komplexes spielt eine entscheidende Rolle. Nach Gabe lineare KM wird ein sehr kleiner Teil des Gd in vivo freigesetzt und bindet an Makromoleküle im Gehirn, welche bisher noch nicht weiter charakterisiert wurden. Diese Bindung von Gd an Makromoleküle ist letztendlich die Ursache, dass sehr kleine Mengen von Gd zu einem sichtbaren SI-Anstieg in den Kerngebieten führen.

- Bis jetzt wurden keine histopathologischen Veränderungen im Gehirn nachgewiesen. Dies wiederum stellt den großen Unterschied zur NSF dar, wo Gd-Ablagerungen in Organen, insbesondere der Haut, zu massiven histopathologischen Veränderungen, wie Inflammation und letztendlich Fibrose führte.

Der klinische Nutzen von Gd-haltiger $\mathrm{KM}$ ist seit Jahrzehnten unumstritten. Ob die seit Mitte 2013 berichteten erhöhten SI- und Gd-Werte eine klinische Relevanz haben, ist unbekannt. Die Gesundheitsbehörden haben die vorliegenden Daten unterschiedlich bewertet. Die EK ist den Empfehlungen der EMA gefolgt und hat als Vorsichtsmaßnahme den weiteren Gebrauch der linearen KM, mit Ausnahme der Leberbildgebung mit Gadoxetsäure (Primovist ${ }^{\circledR}$ ) und Gadobensäure (MultiHance ${ }^{\circledR}$ ) sowie der intraartikulären Anwendung von Ga- dopentetsäure (Magnevist ${ }^{\circledR}$ ) untersagt. Der Gebrauch der makrozyklischen KM ist weiterhin uneingeschränkt möglich. Die meisten anderen Gesundheitsbehörden haben die Hersteller aufgefordert, entsprechende Warnhinweise in den Packungsbeilagen zu ergänzen, die USamerikanische FDA fordert weitere präklinische und klinische Studien.

\section{Sonstige unspezifische Symptome}

Neben den Berichten über Gd im Gehirn und anderen Geweben sowie bildbasierten Befunden von erhöhten SI in bestimmten Hirnarealen nach wiederholter Gabe linearer KM, gibt es Diskussionen um einen Aspekt, der davon deutlich unterschieden werden muss.

In letzter Zeit häufen sich Berichte von Patienten, bei denen noch lange nach einer KM-verstärkten MR-Untersuchung, Gd im Urin, Serum, Haaren und Fingernägeln gefunden wurde. Ein Teil der Patienten berichtet darüber hinaus über unspezifische Beschwerden, wie Kopfschmerzen, Schmerzen und Brennen in den Extremitäten sowie Hautverdickungen. Die Berichte betreffen alle Gd-haltigen KM gleichermaßen, unabhängig von ihrer Struktur und Stabilität. Von Semelka et al. wurde die Hypothese formuliert, dass die Beschwerden auf Gd zurückzuführen seien [54].

Es muss an dieser Stelle ausdrücklich betont werden, dass bislang kein kausaler Zusammenhang der aufgetretenen Beschwerden und der KM-Gabe hergestellt werden konnte. Solange aber keine umfangreichen klinischen Untersuchungen und Daten von entsprechenden Studien vorliegen, kann man es grundsätzlich auch nicht ausschließen. Geringe Fallzahlen und überproportional viele Patienten mit Autoimmunerkrankungen könnten ein Hinweis darauf sein, dass möglicherweise spezifische Dispositionen verantwortlich sind.

\section{Fazit für die Praxis}

\footnotetext{
- Es sind keine Nebenwirkungen bekannt, die kausal auf das Vorhandensein von Gd im Gehirn zurückführen sind.
}

\section{- Bei gegebener klinischer Indikation gibt es keinen bekannten medizi- nischen Grund, einem Patienten die Anwendung Gd-basierter KM vorzuenthalten. \\ - Eine KM-Gabe sollte jedoch immer das Ergebnis einer positiven, indi- viduellen Nutzen-Risiko-Bewertung sein. Besonders sorgfältig sollten diese bei Patienten, die wiederholt KM erhalten müssen (insbesondere bei Kindern) erfolgen. \\ - Aufgabe der wissenschaftlicher Fach- gesellschaften und Verbände sollte es sein, die Leitlinien dahingehend zu überprüfen, ob die empfohlenen Abstände von Wiederholungsunter- suchungen mit KM in der bisherigen Form zu rechtfertigen sind.}

\section{Korrespondenzadresse

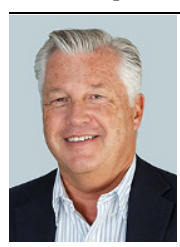 \\ T. Balzer, MD \\ Pharmaceuticals, Medical \& Clinical Affairs Radiology, Bayer U.S. LLC 100 Bayer Boulevard, 07981 Whippany, NJ, USA thomas.balzer@bayer.com}

\section{Einhaltung ethischer Richtlinien}

Interessenkonflikt. L. Schöckel, T. Balzer und H. Pietsch sind Mitarbeiter der Bayer AG.

Alle nationalen Richtlinien zur Haltung und zum Umgang mit Labortieren wurden eingehalten. Die notwendigen Zustimmungen der zuständigen Behörden liegen vor.

Open Access. Dieser Artikel wird unter der Creative Commons Namensnennung 4.0 International Lizenz (http://creativecommons.org/licenses/by/4.0/deed. de) veröffentlicht, welche die Nutzung, Vervielfältigung, Bearbeitung, Verbreitung und Wiedergabe in jeglichem Medium und Format erlaubt, sofern Sie den/die ursprünglichen Autor(en) und die Quelle ordnungsgemäßnennen, einen Link zur Creative Commons Lizenz beifügen und angeben, ob Änderungen vorgenommen wurden.

\section{Literatur}

1. Kanda T, Ishii K, Kawaguchi H et al (2014) High signal intensity in the dentate nucleus and globus pallidus on unenhanced T1-weighted MR images: relationship with increasing cumulative dose of a gadolinium-based contrast material. Radiology 270:834-841

2. Radbruch A, Weberling LD, Kieslich PJ et al (2015) Gadolinium retention in the dentate nucleus and 
globus pallidus is dependent on the class of contrastagent. Radiology 275:783-791

3. Weberling LD, Kieslich PJ, Kickingereder $P$ et al (2015) Increased signal intensity in the dentate nucleus on unenhanced T1-weighted images after gadobenate dimeglumine administration. Invest Radiol 50:743-748

4. Errante Y, Cirimele V, Mallio CA et al (2014) Progressive increase of $\mathrm{T} 1$ signal intensity of the dentate nucleus on unenhanced magnetic resonance images is associated with cumulative doses of intravenously administered gadodiamide in patients with normal renal function, suggesting dechelation. Invest Radiol 49:685-690

5. Quattrocchi CC, Mallio CA, Errante Y et al (2015) Gadodiamide and dentate nucleus T1 hyperintensity in patients with meningioma evaluated by multiple follow-up contrast-enhanced magnetic resonance examinations with no systemic interval therapy. Invest Radiol 50:470-472

6. Ichikawa S, Motosugi U, Omiya Y et al (2017) Contrast agent-induced high signal intensity in dentate nucleus on unenhanced T1-weighted images: comparison of gadodiamide and gadoxetic acid. Invest Radiol 52:389-395

7. Conte G, Preda L, Cocorocchio E et al (2017) Signal intensity change on unenhanced $\mathrm{T} 1$-weighted images in dentate nucleus and globus pallidus after multiple administrations of gadoxetate disodium: an intraindividual comparative study. Eur Radiol 27:4372-4378

8. Kahn J, Posch H, Steffen IG et al (2017) Is there long-term signal intensity increase in the central nervous system on T1-weighted images after MR imaging with the hepatospecific contrast agent gadoxetic acid? A cross-sectional study in 91 patients. Radiology. https://doi.org/10.1148/ radiol.2016162535:162535

9. Yoo RE, Sohn CH, Kang KM et al (2017) Evaluation of gadolinium retention after serial administrations of a macrocyclic gadolinium-based contrast agent (gadobutrol): a single-institution experience with 189 patients. Invest Radiol. https://doi.org/10. 1097/RLI.0000000000000404

10. Radbruch A, Haase R, Kieslich PJ et al (2016) No signal intensity increase in the dentate nucleus on unenhanced T1-weighted MR images after more than 20 serial injections of macrocyclic gadolinium-based contrast agents. Radiology. https://doi.org/10.1148/radiol.2016162241

11. Lee JY, Park JE, Kim HS et al (2017) Up to 52 administrations of macrocyclic ionic MR contrast agent are not associated with intracranial gadolinium deposition: multifactorial analysis in 385 patients. PLoSONE 12:e183916

12. Stojanov DA, Aracki-Trenkic A, Vojinovic $S$ et al (2016) Increasing signal intensity within the dentate nucleus and globus pallidus on unenhanced T1W magnetic resonance images in patients with relapsing-remitting multiple sclerosis: correlation with cumulative dose of a macrocyclic gadolinium-based contrast agent, gadobutrol. Eur Radiol 26:807-815

13. Rossi Espagnet MC, Bernardi B, Pasquini $L$ et al (2017) Signal intensity at unenhanced T1-weighted magnetic resonance in the globus pallidus and dentate nucleus after serial administrations of a macrocyclic gadolinium-based contrast agent in children. Pediatr Radiol. https://doi.org/10.1007/ s00247-017-3874-1

14. Bjornerud A, Vatnehol SAS, Larsson C et al (2017) Signal enhancement of the dentate nucleus at unenhanced MR imaging after very high cumulative doses of the macrocyclic gadolinium- based contrast agent gadobutrol:an observational study. Radiology 285:434-444

15. Splendiani A, Perri M, Marsecano C et al (2017) Effects of serial macrocyclic-based contrast materials gadoterate meglumine and gadobutrol administrations on gadolinium-related dentate nuclei signal increases in unenhanced T1weighted brain: a retrospective study in 158 multiple sclerosis (MS) patients. Radiol Med. https://doi.org/10.1007/s11547-017-0816-9

16. Agris J, Pietsch H, Balzer T (2016) What evidence is there that gadobutrol causes increasing signal intensity within the dentate nucleus and globus pallidus on unenhanced T1W MRI in patients with RRMS? Eur Radiol 26:816-817

17. Lancelot E, Raynaud JS, Desche P (2017) Lack of evidence of a relationship between magnetic resonance signal intensity changes in the globus pallidus and dentate nucleus, and repeated administrations of gadoterate meglumine in children. Pediatr Radiol. https://doi.org/10.1007/ s00247-017-3947-1

18. Radbruch A, Quattrocchi CC (2017) Interpreting signal-intensity ratios without visible $\mathrm{T} 1$ hyperintensities in clinical gadolinium retention studies. Pediatr Radiol 47:1688-1689

19. Stojanov DA (2016) Reply to letter to the editor re: increasing signal intensity within the dentate nucleus and globus pallidus on unenhanced T1W magnetic resonance images in patients with relapsing-remitting multiple sclerosis: correlation with cumulative dose of a macrocyclic gadoliniumbased contrast agent, gadobutrol. Eur Radiol 26:818-819

20. Rossi Espagnet MC, Toma P, Napolitano A (2017) Reply to Radbruch et al.: 'interpreting signalintensity ratios without visible T1 hyperintensities in clinical gadolinium retention studies'. Pediatr Radiol 47:1690-1691

21. Rossi Espagnet MC, Toma P, Napolitano A (2017) Reply to Lancelot et al.: 'Lack of evidence of a relationship between magnetic resonance signal intensity changes in the globus pallidus and dentate nucleus, and repeated administrations of gadoterate meglumine in children'. Pediatr Radiol 47:1694-1696

22. McDonald JS, McDonald RJ, Jentoft ME et al (2017) Intracranial gadolinium deposition following gadodiamide-enhanced magnetic resonance imaging in pediatric patients: a case-control study. JAMA Pediatr. https://doi.org/10.1001/ jamapediatrics.2017.0264

23. McDonald RJ, McDonald JS, Kallmes DF et al (2015) Intracranial gadolinium deposition after contrastenhanced MR imaging. Radiology 275:772-782

24. McDonald RJ, McDonald JS, Kallmes DF et al (2017) Gadolinium deposition in human brain tissues after contrast-enhanced MR imaging in adult patients without Intracranial abnormalities. Radiology. https://doi.org/10.1148/radiol.2017161595: 161595

25. Roberts DR, Welsh CA, LeBel DP 2nd et al (2017) Distribution map of gadolinium deposition within the cerebellum following GBCA administration. Neurology 88:1206-1208

26. Murata N, Gonzalez-Cuyar LF, Murata Ket al (2016) Macrocyclic and other non-group 1 gadolinium contrast agents deposit low levels of gadolinium in brain and bone tissue: preliminary results from 9 patients with normal renal function. Invest Radiol. https://doi.org/10.1097/RLI.0000000000000252

27. Kanda T, Fukusato T, Matsuda M et al (2015) Gadolinium-based contrast agent accumulates in the brain even in subjects without severe renal dysfunction: evaluation of autopsy brain specimens with inductively coupled plasma mass spectroscopy. Radiology 276:228-232

28. Fingerhut S, Niehoff AC, Sperling M et al (2018) Spatially resolved quantification of gadolinium deposited in the brain of a patient treated with gadolinium-based contrast agents. J Trace Elem MedBiol 45:125-130

29. Fingerhut $S$, Sperling $M$, Holling $M$ et al (2018) Gadolinium-based contrast agents induce gadolinium deposits in cerebral vessel walls, while the neuropil is not affected: an autopsy study. Acta Neuropathol 136:127-138

30. Gibby WA, Gibby KA, Gibby WA (2004) Comparison of Gd DTPA-BMA (Omniscan) versus Gd HP-DO3A (ProHance) retention in human bone tissue by inductively coupled plasma atomic emission spectroscopy. Invest Radiol 39:138-142

31. Darrah TH, Prutsman-Pfeiffer JJ, Poreda RJ et al (2009) Incorporation of excess gadolinium into human bone from medical contrast agents. Metallomics 1:479-488

32. Roberts DR, Lindhorst SM, Welsh CT et al (2016) High levels of gadolinium deposition in the skin of a patient with normal renal function. Invest Radiol 51:280-289

33. Frenzel $T$, Lengsfeld $P$, Schirmer $H$ et al (2008) Stability of gadolinium-based magnetic resonance imaging contrast agents in human serum at 37 degrees C. Invest Radiol 43:817-828

34. Schmitt-Willich H (2007) Stability of linear and macrocyclic gadolinium based contrast agents. $\mathrm{Br}$ JRadiol 80:581-582 (author reply 584-585)

35. Sieber MA, Pietsch $H$, Walter J et al (2008) A preclinical study to investigate the development of nephrogenic systemic fibrosis: a possible role for gadolinium-based contrast media. Invest Radiol 43:65-75

36. Jost G, Lenhard DC, Sieber MA et al (2016) Signal increase on unenhanced T1-weighted images in the rat brain after repeated, extended doses of gadolinium-based contrast agents: comparison of linear and macrocyclic agents. Invest Radiol 51:83-89

37. Robert P, Lehericy S, Grand S et al (2015) T1weighted hypersignal in the deep cerebellar nuclei after repeated administrations of gadoliniumbased contrast agents in healthy rats: difference between linear and macrocyclic agents. Invest Radiol 50:473-480

38. Robert P, Violas X, Grand S et al (2016) Linear gadolinium-based contrast agents are associated with brain gadolinium retention in healthy rats. Invest Radiol 51:73-82

39. Robert $P$, Fingerhut $S$, Factor C etal (2018) One-year retention of gadolinium in the brain: comparison of gadodiamide and gadoterate meglumine in a rodent model. Radiology 288:424-433

40. Jost G, Frenzel T, Boyken J et al (2018) Longterm excretion of gadolinium-based contrast agents: linear versus macrocyclic agents in an experimental rat model. Radiology. https://doi. org/10.1148/radiol.2018180135:180135

41. Robert P, Fingerhut S, Factor C et al (2018) One-year retention of gadolinium in the brain: comparison of gadodiamide and gadoterate meglumine in a rodent model. Radiology. https://doi.org/10. 1148/radiol.2018172746:172746

42. McDonald RJ, McDonald JS, Dai D et al (2017) Comparison of gadolinium concentrations within multiple rat organs after intravenous administration of linear versus macrocyclic gadolinium chelates. Radiology. https://doi.org/10.1148/ radiol.2017161594:161594 
43. Boyken J, Niendorf T, Flemming B et al (2018) Gadolinium deposition in the brain after contrastenhanced MRI: Are the data valid? Radiology. https://doi.org/10.1148/radiol.2018171762: 171762

44. Idee JM, Robert P, Raynaud JS et al (2018) Region of interest selection in nonclinical studies of accumulated gadolinium-based contrast agentinduced T1 hyperintensity in deep cerebellar nuclei. Radiology 287:360-362

45. Rasschaert M, Idee JM, Robert P et al (2017) Moderate renal failure accentuates $\mathrm{T} 1$ signal enhancement in the deep cerebellar nuclei of gadodiamide-treated rats. Invest Radiol 52:255-264

46. Kartamihardja AA, Nakajima T, Kameo Set al (2016) Impact of impaired renal function on gadolinium retention after administration of gadoliniumbased contrast agents in a mouse model. Invest Radiol 51:655-660

47. Jost G, Frenzel T, Lohrke J et al (2016) Penetration and distribution of gadolinium-based contrast agents into the cerebrospinal fluid in healthy rats: a potential pathway of entry into the brain tissue. Eur Radiol. https://doi.org/10.1007/s00330-0164654-2

48. Jessen NA, Munk AS, Lundgaard I et al (2015) The glymphatic system: a beginner's guide. Neurochem Res 40:2583-2599

49. Deike-Hofmann K, Reuter J, Haase R et al (2018) Glymphatic pathway of gadolinium-based contrast agents through the brain: overlooked and misinterpreted. Invest Radiol. https://doi.org/10. 1097/RLI.0000000000000533

50. Jost G, Frenzel T, Boyken J et al (2018) Impact of brain tumors and radiotherapy on the presence of gadolinium in the brain after repeated administration of gadolinium based contrast agents: an experimental study on rats. RSNA, Chicago

51. Frenzel T, Apte C, Jost G et al (2017) Quantification and assessment of the chemical form of residual gadolinium in the brain after repeated administration of gadolinium-based contrast agents: comparative study in rats. Invest Radiol. https:// doi.org/10.1097/RLI.0000000000000352

52. Lohrke J, Frenzel T, Schöckel L et al (2017) Histology and gadolinium distribution in the rodent brain after the administration of cumulative high doses of linear and macrocyclic gadolinium-based contrast agents. Invest Radiol. https://doi.org/10. 1097/RLI.0000000000000344

53. Smith AP, Marino M, Roberts J et al (2017) Clearance of gadolinium from the brain with no pathologic effect after repeated administration of gadodiamide in healthy rats: an analytical and histologic study. Radiology 282:743-751

54. Semelka RC, Ramalho J, Vakharia A et al (2016) Gadolinium deposition disease: initial description of a disease that has been around for a while. Magn Reson Imaging 34:1383-1390

\section{Erratum}

Radiologe 2019.59:368

https://doi.org/10.1007/s00117-019-0518-5

Online publiziert: 21. März 2019

(c) Springer Medizin Verlag GmbH, ein Teil von Springer Nature 2019

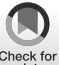

Check for
updates

\section{H. Kopf' $\cdot$ W. Schima' $\cdot$ S. Meng ${ }^{2,3}$}

${ }^{1}$ Abteilung für Diagnostische und Interventionelle Radiologie, Göttlicher Heiland Krankenhaus, Vinzenzgruppe, Wien, Österreich

${ }^{2}$ Radiologie, KFJ Spital, Wien, Österreich

${ }^{3}$ Zentrumfür Anatomie und Zellbiologie, Medizinische Universität Wien, Wien, Österreich

\section{Erratum zu: Differenzialdiagnose von Befunden an der Gallenblase}

\section{Ultraschall, Computertomographie und Magnetresonanztomographie}

\section{Erratum zu:}

Radiologe 2019

https://doi.org/10.1007/s00117-019-

0504-y

In der Online-Version dieses Beitrags wurde leider die Abb. 6 fehlerhaft wiedergegeben. Der Originalartikel wurde korrigiert - wir bitten um Beachtung.

\section{Korrespondenzadresse}

\section{PD Dr. S. Meng}

Zentrumfür Anatomie und Zellbiologie, Medizinische Universität Wien Währinger Str. 13, 1090 Wien, Österreich stefan.meng@muv.ac.at
Die Online-Version des Originalartikels ist unter https://doi.org/10.1007/s00117-019-0504-y zu finden. 